\title{
Stellar-mass black holes in star clusters: implications for gravitational-wave radiation
}

\author{
Sambaran Banerjee, ${ }^{1,2}$ Holger Baumgardt ${ }^{1}$ and Pavel Kroupa ${ }^{1}$ \\ ${ }^{1}$ Argelander-Institut für Astronomie, Auf dem Hügel 71, 53121, Bonn, Germany \\ email: [sambaran, holger, pavel] @astro.uni-bonn.de \\ ${ }^{2}$ Alexander von Humboldt fellow
}

\begin{abstract}
We study the dynamics of stellar-mass black holes (BHs) in star clusters, with particular attention to the formation of $\mathrm{BH}-\mathrm{BH}$ binaries, which are interesting as sources of gravitational waves (GWs). We examine the properties of these $\mathrm{BH}-\mathrm{BH}$ binaries through direct $N$-body simulations of Plummer clusters of $N \leqslant 10^{5}$ low-mass stars with an initial population of stellar-mass BHs, using the NBODY6 code. We find that the stellar-mass BHs segregate rapidly into the cluster core and form a dense subcluster of $\mathrm{BHs}$ in which $\mathrm{BH}-\mathrm{BH}$ binaries form through three-body encounters. While most $\mathrm{BH}$ binaries are ejected from the cluster by recoils due to superelastic encounters with the single BHs, we find that for clusters with $N \gtrsim 5 \times 10^{4}$, typically a few of them harden sufficiently so that they can merge via GW emission within the cluster. Also, for each of such clusters there are a few escaping BH binaries that merge within a Hubble time, with most merger times being within a few Gyr. These results imply that the intermediate-age massive clusters constitute the most important class of star cluster candidates that can produce dynamical $\mathrm{BH}-\mathrm{BH}$ mergers at the present epoch. The $\mathrm{BH}-\mathrm{BH}$ merger rates obtained from our computations imply a significant detection rate $\left(\sim 30 \mathrm{yr}^{-1}\right)$ for the proposed Advanced LIGO GW detector.
\end{abstract}

Keywords. gravitational waves, black hole physics, stellar dynamics, scattering, methods: $N$ body simulations, methods: numerical, galaxies: star clusters, binaries: close

\section{Introduction}

Star clusters, e.g., globular clusters (henceforth GCs), young and intermediate-age massive clusters (henceforth IMCs) harbor a large overdensity of compact stellar remnants compared to the field by virtue of their mass segregation. These compact stars, which are neutron stars (henceforth NSs) and black holes (henceforth BHs), are produced by stellar evolution of the most massive stars of the cluster within the first $\sim 50 \mathrm{Myr}$ after cluster formation. Since they are heavier than the remaining low-mass stars in the cluster, they segregate quickly (within one or a few half-mass relaxation times) to the cluster core, forming a dense subcluster of compact stars. Such a compact stellar subcluster is of wide interest as it is efficient in producing compact-star binaries dynamically, which are of interest for a wide range of physical phenomena, e.g., X-ray emission (Xray binaries), gravitational waves ( $\mathrm{BH}-\mathrm{BH}$ and $\mathrm{NS}-\mathrm{NS}$ binaries) and short gamma-ray bursts (NS-NS and NS-BH binaries). In the present work, we investigate the dynamics of stellar-mass BHs in star clusters, which are formed through supernova explosions of $\gtrsim 18 \mathrm{M}_{\odot}$ stars, typically within the mass range $8 \mathrm{M}_{\odot} \lesssim M_{\mathrm{BH}} \lesssim 12 \mathrm{M}_{\odot}$ for solar-metallicity progenitors (Casares 2007). We are particularly interested in the dynamically formed $\mathrm{BH}-\mathrm{BH}$ binaries, which are strong sources of GWs as they spiral in via GW radiation.

As studied earlier by several authors (e.g., Merritt et al. 2006; Mackey et al. 2007), $\mathrm{BHs}$, formed through stellar evolution, segregate into the cluster core within $\sim 0.3 \mathrm{pc}$ 
and form a dynamically isolated subcluster of BHs, where the density of BHs is large enough that $\mathrm{BH}-\mathrm{BH}$ binary formation through three-body encounters (Heggie \& Hut 2003) becomes important. These dynamically formed BH binaries then 'harden' through repeated superelastic encounters with the surrounding BHs (Heggie's law; Heggie 1975). The binding energy of the $\mathrm{BH}$ binaries released is carried away by the single $\mathrm{BHs}$ and $\mathrm{BH}$ binaries involved in the encounters. This causes the $\mathrm{BHs}$ and $\mathrm{BH}$ binaries to get ejected from the $\mathrm{BH}$ core to larger radii in the cluster, and they deposit energy to the cluster stars while sinking back to the core through dynamical friction, thereby heating the cluster. As the BH binaries harden, the encounter-driven recoil becomes stronger and finally the recoil is large enough that the encountering single $\mathrm{BH}$ and/or the $\mathrm{BH}$ binary escapes from the cluster. This also results in cluster heating because of the associated mass loss from the cluster core that decreases its potential energy correspondingly. These heating mechanisms result in cluster expansion.

In the present work, we make a detailed study of the dynamics of $\mathrm{BH}-\mathrm{BH}$ binaries formed in a $\mathrm{BH}$ subcluster. In particular, we investigate whether hard enough $\mathrm{BH}$ binaries that can merge via gravitational radiation in a Hubble time within the cluster or after getting ejected, can be formed in such a subcluster. To that end, we perform a series of $N$-body integrations of concentrated star clusters (half-mass radius $r_{\mathrm{h}} \leqslant 1$ pc) consisting of $\leqslant 10^{5}$ low-mass stars, to which a certain number of stellar-mass BHs is added, representing a star cluster with an evolved stellar population.

\section{Computations}

To study the dynamics of BHs in star clusters, we perform direct $N$-body simulations with star clusters in which a certain number of BHs are added initially, using the NBODY6 code on graphical-processing-unit (GPU) platforms (see Aarseth 2009). The initial cluster follows a Plummer model with half-mass radius $r_{\mathrm{h}} \leqslant 1 \mathrm{pc}$, consisting of $N \leqslant 10^{5}$ lowmass main-sequence stars in the mass-range $0.5 \mathrm{M}_{\odot} \leqslant m \leqslant 1.0 \mathrm{M}_{\odot}$. A specified number of $\mathrm{BHs}$ are added to each cluster with the same initial distribution as the stars. The initial number of $\mathrm{BHs}$ are chosen according to the $\mathrm{BH}$ retention fraction, where we explore both full retention and the case where half of the BHs are ejected from the cluster by natal kicks. For simplicity, we consider only equal-mass BHs in the present work, with the representative value of $M_{\mathrm{BH}}=10 \mathrm{M}_{\odot}$. With such a cluster, we mimic the epoch at which the massive stars have already evolved to produce their remnant BHs.

To evolve the $\mathrm{BH}-\mathrm{BH}$ binaries due to GW emission, the well-known Peters' formula (Peters 1964) is used in NBODY6, according to which the merger time $t_{\mathrm{mrg}}$ of an equalmass $\mathrm{BH}-\mathrm{BH}$ binary due to $\mathrm{GW}$ emission is given by

$$
t_{\mathrm{mrg}}=150 \operatorname{Myr}\left(\frac{\mathrm{M}_{\odot}}{M_{\mathrm{BH}}}\right)^{3}\left(\frac{a}{\mathrm{R}_{\odot}}\right)^{4}\left(1-e^{2}\right)^{7 / 2},
$$

where $a$ is the semimajor axis of the binary and $e$ is its eccentricity. Numerical simulations of $\mathrm{BH}-\mathrm{BH}$ mergers indicate that for unequal-mass BHs or even for equal-mass BHs with unequal spins, the merged $\mathrm{BH}$ product acquires a velocity kick of typically $100 \mathrm{~km} \mathrm{~s}^{-1}$ or more, due to asymmetry in momentum outflow from the system, associated with the GW emission. Therefore, in our computations, we provide an arbitrarily large velocity kick of $150 \mathrm{~km} \mathrm{~s}^{-1}$ immediately after a $\mathrm{BH}-\mathrm{BH}$ merger, to make sure that the merged $\mathrm{BH}$ escapes. Also, we evolve the clusters isolated, i.e., in absence of a galactic tidal field as the formation of the $\mathrm{BH}$ core through mass segregation and its dynamics remain largely unaffected by the presence of a tidal field, which mainly affects stars near the 
Table 1. Summary of the computations performed for isolated clusters and those with reflective boundary (see Section 2). The meaning of different columns is as follows: (1): Identity of the particular model; similar values with different names (ending with A, B, etc.) imply runs repeated with different random seeds. (2): Total number of stars, N. (3): Number of simulations, $N_{\text {sim }}$, with the particular cluster. (4): Initial half-mass radius of the cluster, $r_{\mathrm{h}}(0)$ (isolated cluster), or radius of reflective sphere, $R_{\mathrm{s}}$. (5): Initial number of $\mathrm{BHs}, N_{\mathrm{BH}}(0)$. (6): Total number of $\mathrm{BH}-\mathrm{BH}$ binary mergers within the cluster, $N_{\mathrm{mrg}}$. (7): Times, $t_{\mathrm{mrg}}$, corresponding to the mergers. (8): Number of escaped BH pairs, $N_{\text {esc }}$; the three values of $N_{\text {esc }}$ are for $t_{\mathrm{mrg}}<3 \mathrm{Gyr}, 1 \mathrm{Gyr}$ and 100 Myr, respectively.

\begin{tabular}{|c|c|c|c|c|c|c|c|}
\hline Model name & $N$ & $N_{\text {sim }}$ & $r_{\mathrm{h}}(0)$ or $R_{\mathrm{s}}(\mathrm{pc})$ & $N_{\mathrm{BH}}(0)$ & $N_{\mathrm{mrg}}$ & $t_{\mathrm{mrg}}(\mathrm{Myr})$ & $N_{\text {esc }}$ \\
\hline \multicolumn{8}{|c|}{ Isolated clusters } \\
\hline C10K20 & 10000 & 10 & 1.0 & 20 & 0 & - & - \\
\hline $\mathrm{C} 25 \mathrm{~K} 50$ & 25000 & 10 & 1.0 & 50 & 0 & -- & 311 \\
\hline C50K80 & 45000 & 1 & 1.0 & 80 & 1 & 698.3 & 310 \\
\hline C50K80.1 & 45000 & 1 & 0.5 & 80 & 2 & $217.1,236.6$ & 321 \\
\hline C50K40.1 & 45000 & 1 & 0.5 & 40 & 0 & $-\ldots$ & 111 \\
\hline C50K200 & 50000 & 1 & 1.0 & 200 & 2 & $100.8,467.8$ & 000 \\
\hline C65K110 & 65000 & 1 & 1.0 & 110 & 1 & 314.6 & 421 \\
\hline C65K110.1 & 65000 & 1 & 0.5 & 110 & 0 & -- & 431 \\
\hline C65K55.1 & 65000 & 1 & 0.5 & 55 & 1 & 160.5 & 100 \\
\hline C100K80 & 100000 & 1 & 1.0 & 80 & 2 & $219.4,603.2$ & 521 \\
\hline C100K200 & 100000 & 1 & 1.0 & 200 & 0 & -- & 544 \\
\hline \multicolumn{8}{|c|}{ Reflective boundary } \\
\hline R3K180 & 3000 & 1 & 0.4 & 180 & 1 & 1723.9 & 531 \\
\hline R4K180A & 4000 & 1 & 0.4 & 180 & 1 & 3008.8 & 221 \\
\hline R4K180B & 4000 & 1 & 0.4 & 180 & 2 & $100.2,1966.5$ & 210 \\
\hline R3K100 & 3000 & 1 & 0.4 & 100 & 2 & $3052.8,3645.9$ & 110 \\
\hline R4K100A & 4000 & 1 & 0.4 & 100 & 2 & $104.4,814.2$ & 331 \\
\hline R4K100B & 4000 & 1 & 0.4 & 100 & 1 & 1135.3 & 333 \\
\hline
\end{tabular}

tidal boundary. Further, for simplicity, we do not take into account primordial binaries in this initial study.

\subsection{Simulation of a $G C$ core: reflective boundary}

We also perform integrations with a smaller number of stars and BHs that are confined within a reflecting spherical boundary (Banerjee et al. 2009). With such a dynamical system, one can mimic the core of a massive cluster, where the BHs are concentrated after mass segregation. The advantage of this approach is that one can compute the evolution of a massive cluster with many fewer stars, allowing much faster computation. We integrate $N=3000-4000$ stars, confined within $0.4 \mathrm{pc}$, which provides a stellar density of $\sim 10^{4} \mathrm{M}_{\odot} \mathrm{pc}^{-3}$, appropriate for the core density of a massive cluster. However, stars and BHs faster than a pre-assigned speed, $v_{\mathrm{esc}} \approx 24 \mathrm{~km} \mathrm{~s}^{-1}$, representing the escape speed of the parent cluster, are allowed to escape through the reflective boundary.

\section{Results}

Table 1 summarizes the results of our computations. For demonstration purposes, we use one of the models, C50K80 (see Table 1). Other models generally possess similar properties. Figure 1 (left panel) demonstrates the mass segregation of the BHs in the cluster, which takes about 50 Myr. As the BHs segregate within about $0.3 \mathrm{pc}$ of the 

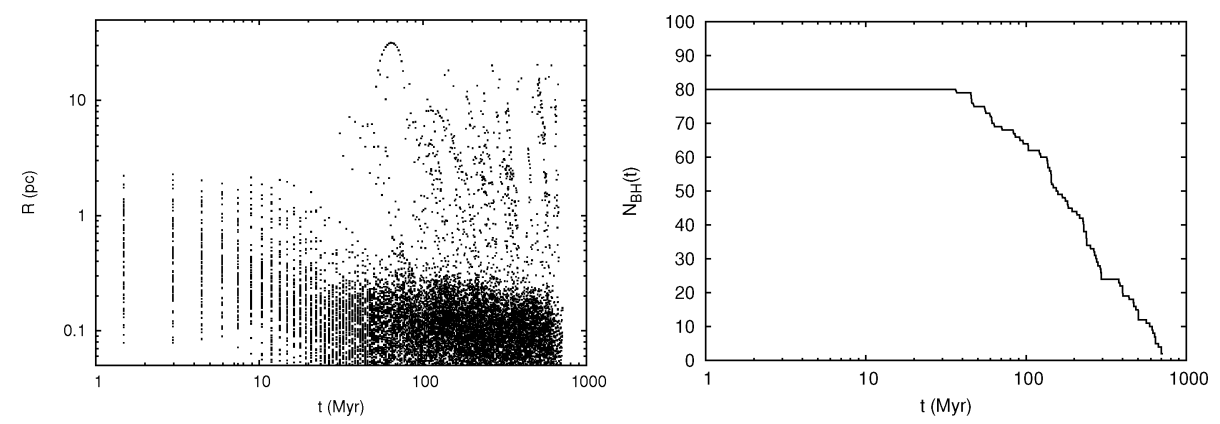

Figure 1. Mass segregation of BHs shown by the radial position $R$ versus time $t$ (left panel) for model C50K80. The BHs segregate in $\sim 50 \mathrm{Myr}$, during which $N_{\mathrm{BH}}$ (the number of BHs bound to the cluster) remains unchanged (right panel). As the $\mathrm{BH}$ subcluster becomes dense enough that $\mathrm{BH}-\mathrm{BH}$ binaries begin to form through three-body encounters, single $\mathrm{BHs}$ and $\mathrm{BH}$ binaries are ejected from the cluster, resulting in a decrease of $N_{\mathrm{BH}}$.

cluster core, the $\mathrm{BH}$ density of this subcluster becomes high enough to form $\mathrm{BH}-\mathrm{BH}$ binaries through three-body encounters. Once BH-BH binaries start forming, single BHs and $\mathrm{BH}$ binaries begin to escape from the $\mathrm{BH}$ core due to encounter recoils (see Section 1). In Figure 1 (left panel), one can clearly distinguish the two phases of the BH subsystem, the initial segregation phase and $\mathrm{BH}$-core formation, the radial positions of the $\mathrm{BHs}$ being scattered outwards in the latter phase due to the recoils. The resulting decrease of $N_{\mathrm{BH}}$ during this phase is also shown in Figure 1 (right panel).

\subsection{Mergers and escapers}

To study the possibility of $\mathrm{BH}-\mathrm{BH}$ mergers, we consider the positions of the $\mathrm{BH}-\mathrm{BH}$ binaries within the cluster in an $a$ versus $\left(1-e^{2}\right)$ plane, as shown in Figure 2, where each pair is represented by a different symbol and color coded with the evolution time in Myr. For each BH pair, $a$ and $e$ fluctuate over the plane, the changes occurring over a collision timescale, of order Myr. Since the orbital periods of the binaries corresponding to these points are much shorter (from $\sim$ days to years), these points generally represent binaries which are stable over many orbits. Overplotted in Figure 2 are lines of constant GW merger time $t_{\mathrm{mrg}}$, as given by Equation (2.1). While most points correspond to very large merger times, a few of them lie close to the $t_{\mathrm{mrg}}=10 \mathrm{Myr}$ line. This indicates that these binaries are indeed hardened up to small enough $a$ and/or acquire sufficient eccentricity that if they are left unperturbed, they can merge via GW emission within several Myr. However, these merging BH pairs can still be perturbed by further encounters on timescales of $\sim$ Myr, which can often prevent them from merging. In the particular example shown in Figure 2, only one of them could merge within the cluster. This feature is found to be generally true for all models reported here (see Table 1), i.e., each produces $\mathrm{BH}$ pairs that are capable of merging within several Myr. On the other hand, among the escaped BH binaries, all of them with GW merger times of less than a Hubble time are of interest, since they remain unperturbed afterwards. The positions of the escaped $\mathrm{BH}$ binaries from the model C65K110 are shown in Figure 3 as an interesting illustration.

Figure 4 (left panel) shows the distribution of merger times, $t_{\mathrm{mrg}}$, of $\mathrm{BH}$ binaries that occurred within the clusters, which indicates that $N_{\mathrm{mrg}}$ decreases with $t_{\mathrm{mrg}}$. This can be expected, as at later times $N_{\mathrm{BH}}$ in a cluster decreases, decreasing the hardening rate of $\mathrm{BH}$ binaries correspondingly. Figure 4 (right panel) shows the distribution of the merger times for the escapers, which also shows a decrease with time, as can be expected from Equation (2.1). The above merger-time distributions indicate that most mergers occur within the first few Gyr of cluster evolution. 


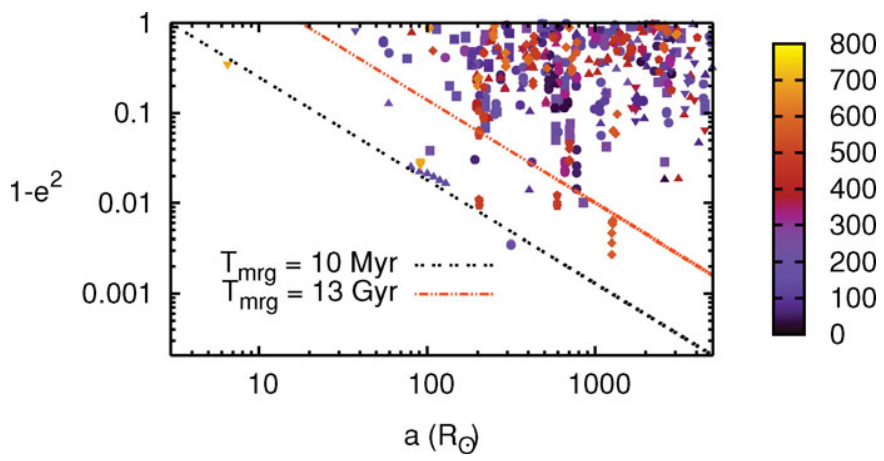

Figure 2. Positions of all BH binaries within the cluster on the $1-e^{2}$ versus $a$ plane, where different symbols are used to distinguish between different BH pairs (for model C50K80). The color coding of the points, as indicated by the color scale, represents the time (in Myr) at which they appear at a particular location in the above plane. Lines of constant GW-merger times $t_{\mathrm{mrg}}=$ constant are overplotted.

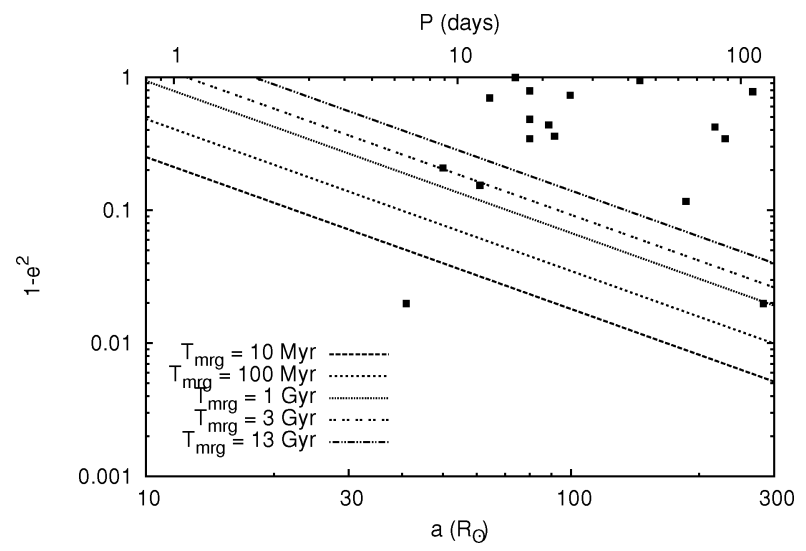

Figure 3. Positions of all escaped $\mathrm{BH}$ binaries in the $1-e^{2}$ versus a plane for model C65K110. Lines of constant merger times are overplotted, as in Figure 2.

These results imply that an important class of candidates for dynamically forming $\mathrm{BH}$ binaries that merge at the present epoch are the star clusters with initial mass $M_{\mathrm{cl}} \gtrsim 3 \times 10^{4} \mathrm{M}_{\odot}$, which are few Gyr old. Such clusters represent IMCs with initial masses close to the upper limit of the initial cluster mass function in spiral and starburst galaxies (Weidner et al. 2004; Gieles et al. 2006; Larsen 2009). Old GCs, which can be about 10 times more massive, are typically much older $(\sim 10 \mathrm{Gyr})$, so that they cannot contribute significantly to the present-day merger rate, as most of their mergers would have occurred much earlier. On the other hand, young massive clusters with ages younger than $50 \mathrm{Myr}$ are generally too young to produce $\mathrm{BH}-\mathrm{BH}$ mergers, as the segregation of $\mathrm{BHs}$ and the formation of the $\mathrm{BH}$ core itself takes longer. Hence, IMCs seem to be the most likely star clusters for dynamically producing present-day $\mathrm{BH}-\mathrm{BH}$ mergers.

\subsection{Detection rate of $B H-B H$ mergers}

Results from Table 1 give an average merger rate of $\approx 0.4$ cluster $^{-1} \mathrm{Gyr}^{-1}$ for $\mathrm{BHs}$ within the clusters and $\approx 0.9$ cluster $^{-1} \mathrm{Gyr}^{-1}$ for the escapers, totalling $\mathcal{R}_{\mathrm{mrg}} \approx 1.3$ cluster $^{-1} \mathrm{Gyr}^{-1}$. To estimate the detection rate of BH-BH mergers from IMCs, we adopt the space density derived by Portegies Zwart \& McMillan (2000) for young populous clusters, $\rho_{\mathrm{cl}} \approx 3.5 h^{3} \mathrm{Mpc}^{-3}$, where $h=0.73$ is the Hubble parameter. The above merger 

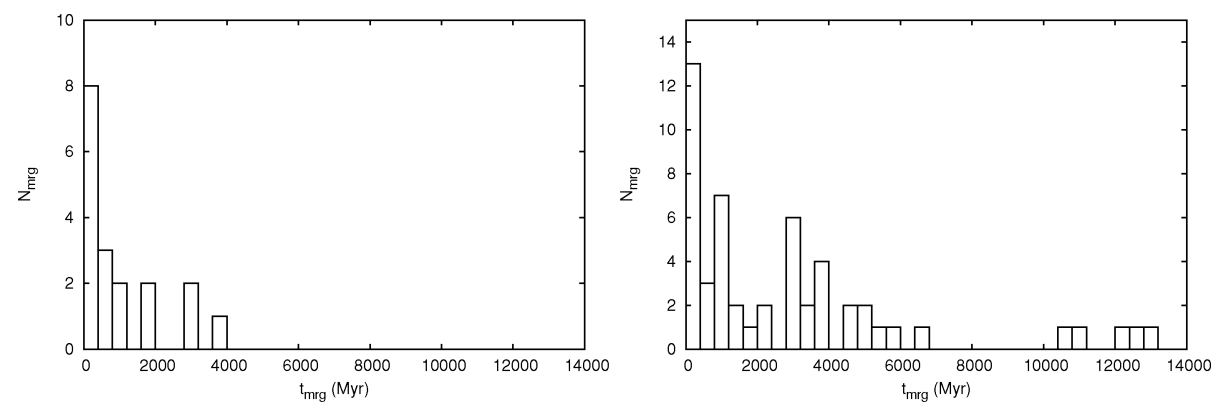

Figure 4. (left) Distribution of the merger times, $t_{\mathrm{mrg}}$, for BH binary mergers within the cluster for the models in Table 1. (right) Distribution of $t_{\mathrm{mrg}}$ for escaped $\mathrm{BH}$ binaries for the models in Table 1.

rate implies (Banerjee et al. 2009) that IMCs would yield a $\mathrm{BH}$-BH-merger detection rate of $\mathcal{R}_{\text {AdLIGO }} \approx 30 \mathrm{yr}^{-1}$ for the future Advanced LIGO (AdLIGO) detector (see, e.g., Belczynski et al. 2007). The present-day LIGO detection rate is, however, negligible, $\mathcal{R}_{\mathrm{LIGO}} \approx 4.6 \times 10^{-3} \mathrm{yr}^{-1}$. Interestingly, the above dynamical $\mathrm{BH}-\mathrm{BH}$-merger detection rate can be more than 10 times higher than that from individual primordial binaries (Belczynski et al. 2007). Our results imply that dynamically formed BH-BH binaries constitute the dominant contribution to $\mathrm{BH}-\mathrm{BH}$ merger detection. Thus, the dynamical $\mathrm{BH}-\mathrm{BH}$ inspirals from star clusters seem to be a promising channel for $\mathrm{GW}$ detection by AdLIGO, although their estimated detection rate with the present LIGO detector is negligible, conforming with the hitherto nondetection of GWs.

\section{Acknowledgements}

We thank the Symposium organizers for selecting this work for presentation. SB acknowledges support from the Alexander von Humboldt Foundation. HB acknowledges support from the German Science Foundation through a Heisenberg fellowship.

\section{References}

Aarseth, S. J. 2009, http://www.sverre.com/

Banerjee, S., Baumgardt, H., \& Kroupa, P. 2009, MNRAS, in press (arXiv:0910.3954)

Belczynski, K., Taam, R. E., Kalogera, V., Rasio, F. A., \& Bulik, T. 2007, ApJ, 662, 504

Casares, J. 2007, in: V. Karas \& G. Matt. (eds.), Black Holes from Stars to Galaxies: Across the Range of Masses, IAU Symp, 238, p. 3

Gieles, M., Larsen, S. S., Scheepmaker, R. A., Bastian, N., Haas, M. R., \& Lamers, H. J. G. L. M. 2006, $A \mathscr{E} A, 446$, L9

Heggie, D. C. 1975, MNRAS, 173, 729

Heggie, D. C. \& Hut, P. 2003, The Gravitational Millon-Body Problem: A Multidisciplinary Approach to Star Cluster Dynamics (Cambridge: Cambridge University Press)

Larsen, S. S. 2009, A\& A, 494, 539

Mackey, A. D., Wilkinson, M. I., Davies, M. B., \& Gilmore, G. F. 2007, MNRAS, 379, L40

Merritt, D., Piatek, S., Portegies Zwart, S., \& Hemsendorf, M. 2004, ApJ, 608, L25

Peters, P. C. 1964, Phys. Rev. B, 136, 1224

Portegies Zwart, S. F. \& McMillan, S. L. W., 2000, ApJ (Letters), 528, L17

Weidner, C., Kroupa, P., \& Larsen, S. S. 2004, MNRAS, 350, 1503 